\title{
"Health Technology Assessment in High, Middle and Low-income Countries: New Systematic and Interdisciplinary Approach for Sound Informed-policymaking"
}

Mohammed AlKhaldi ( $\nabla$ moh.khaldi83@gmail.com )

McGill University https://orcid.org/0000-0001-5609-3806

\section{Sara Ahmed}

McGill University Faculty of Medicine

Aisha Al Basuoni

Gaza Community Mental Health Programme

Marcel Tanner

University of Basel: Universitat Basel

\section{Methodology}

Keywords: HSs, WHO, SDGs, HTA, current HTA practices, application, capacity, gaps

Posted Date: December 10th, 2020

DOI: https://doi.org/10.21203/rs.3.rs-123807/v1

License: @ (1) This work is licensed under a Creative Commons Attribution 4.0 International License. Read Full License 


\section{Abstract}

Technological innovation has a significant role in improving health systems (HSs) and achieving universal health coverage. The World Health Organization (WHO) has declared resolutions on Health Technology Assessment (HTA) and other global organizations emphasized on HTA systems to achieve the Sustainable Development Goals (SDGs). HTA is a modern multidisciplinary decisionmaking framework linking knowledge and policymaking in order to provide evidence to leaders and ensuring the value of resources by evaluating properties, effects, and/or impacts. The scope of HTA focuses on conducting assessments and analyses to investigate the medical, social, economic, organizational and ethical issues within health and social systems for generating management and technical solutions. HTA is important as it is rapidly growing and is seen as an essential development approach to tackle existing challenges, particularly in developing countries as they share most of the health burdens worldwide.

The research aims to comprehensively evaluate HTA within the health and social systems and understand HTA within the national health system with regards to the level of knowledge about HTA, current HTA practices, application, capacity, gaps, and solutions by investigating the perceptions of health systems' stakeholders in five countries, Canada, Switzerland, Lebanon, Palestine, and Tanzania selected according to the World Bank income classification.

The project will last 12 months starts in January 2021 and ends in January 2022. A mixed-methods, quantitative and qualitative, along with a scoping review will be applied. In each country, fifty semi-structured questionnaires, twenty in-depth interviews, and one national focus group discussion will be conducted with health experts, managers, and policymakers selected purposively from the 1st and 2nd levels of the HS structure. Excel, IBM Statistical Package for the Social Sciences (SPSS), and MAXQDA 12 (VERBI GmbH, Berlin) software programs will be used for data management and analysis.

The research will form cutting-edge evidence and reference not only for the six countries, but also for the global, regional, and national endeavors with regards to opening a room for HTA best application and optimization based on the produced knowledge from this research. It will reveal lessons learned, determine gaps, and set an applicable strengthening framework for HTA. This framework will eventually aid the decision and policymakers in these countries, and other similar countries and international organizations to build a well-enabled and institutionalized HTA for better universal health coverage, health systems, and multi-sectoral development.

\section{Background}

Technological innovation has a significant role in improving healthcare delivery and universal health coverage, where the World Health Organization (WHO) and other global health organizations emphasize supporting regional and national Health Technology Assessment (HTA) systems. HTA is immensely increasing not only in developed countries but also in developing countries. It reduces resource waste, inefficiencies, and inappropriate investments in Health Systems (HSs). HTA is a multidisciplinary decision-making process that uses information about the medical, social, economic, organizational and ethical issues related to the use of growing health technologies and interventions such as information and communication technology, E-Health, electronic health records, medical devices, diagnostic devices, clinical interventions, medicines, vaccines, and practice management solutions. Assessing the effectiveness and optimal utilization of these interventions within the health and social systems using sound methodological evaluation, assessments, and analyses like HTA is indispensable before being introduced into practice (Banta, 2003; Ritrovato et al., 2015; WHO, EMRO n.d., n.d.). As it is a framework that bridges research and policymaking, this evaluation provides evidence to decision-makers and ensures the value of money and resources (WHO, 2013; WHO, EMRO, 2014) by evaluating properties, effects, and/or impacts (Marcial Velasco Garrido et al., 2008). HTA may be seen as a development strategic question that needs to be addressed institutionally, nationally, regionally, and globally. Implementing HTA is particularly important to improve health surveillance, HS management, health education, and clinical decision-making, and to support behavioral changes related to public-health priorities and long-term diseases management.

\section{Research Significance And Rational}

Globally, HTA receives great attention and is seen as a development approach. WHO has declared resolutions to adopt HTA as an essential approach to tackle global challenges, improve HSs and achieve the Sustainable Development Goals (SDGs) (Dankó, 2014). The rationale behind conducting this research is that, in developed countries, HTA is rapidly growing, for example, Canada and Switzerland, are two leading countries in this field despite some challenges in their HTA systems independence, methodology, and implementation ("HTA in Switzerland - SwissHTA - Swiss Health Technology Assessment," n.d.; Menon and Stafinski, 2009). As the 
less-resourced developing countries are in more need of HTA, there are considerable unrecognized gaps in HTA understanding, practice, and utilization. Regionally, Figure one reveals that $52 \%$ of the Middle East countries are performing and utilizing HTA or HTAlike activities, which means that $48 \%$ are still not performing HTA-like activities. Furthermore, using HTA in the decision-making process was viewed as the biggest obstacle (WHO, 2015; WHO Regional Office for the Eastern Mediterranean, Cairo, 2015). Healthcare interventions and technologies in the Middle East region, with all its components, innovation, regulation, assessment, and management, remain a major challenge (the Middle East and North Africa Health Policy Forum, 2017). Therefore, Lebanon, Palestine, and Tanzania are essentially in need for HTA to manage the scarcity and misallocation of the resource on one hand, and to tackle many challenges that represent a large proportion of the burden of disease on another hand (Marcial Velasco Garrido et al., 2008; Oortwijn et al., 2010; Rugunda and Asadi-Lari, 2014; WHO, 2017; WHO, EMRO, 2014).

The importance of this research project stems from insufficient knowledge and literature about the HTA in the region and specifically in the three countries, Lebanon, Palestine, and Tanzania. The project will advance the existing evidence on the subject and will generate optimal knowledge translation strategies for useful HTA frameworks and models which is the focus of this project. While in developed countries such as Canada and Switzerland, HTA at the local and provincial levels needs to be assessed for further improvement and importantly transferring the successful adapted models and experience to our developing countries. Therefore, the approach of evaluating HTA from various settings and contexts is useful where it will develop reliable evidence, insights, and comparisons to be added to the current knowledge advancement and for innovating further global and regional best practices of HTA. The ideation of this research emerged from the Principal Investigator's (PI) Ph.D. project, which dealt with analyzing the whole health research system in Palestine targeting three sectors: academic, government, and local and international organizations. Both topics, Health Research System (HRS) and HTA, are organically linked to each other, which both urge for planning and implementing health interventions and making decisions based on research and evidence. The key recommended policy advice was implementing health policy and systems research (HPSR) and using innovative and evidence-based approaches and instruments to support the evidence-informed decisionmaking in the health sector in Palestine (AlKhaldi, 2018). Figure two illustrates that Evidence-informed policy-making for health should be driven by six essential pillars, and HTA is one of them that is required to be assessed and strengthened (WHO, EMRO, n.d.).

Additionally, HTA is addressing such an interdisciplinary approach that incorporates various sectors and disciplines to collaborate together to employ technology, science, and health research to strengthen our HSs and to solve our communities' problems is an optimal goal and crucial demand (Banta, 2003). In this context, investigating HTA systems in the five selected countries may also open a new horizon for fostering SDGs achievement in the five countries with a focus on the three developing countries (Palestine, Lebanon, and Tanzania).

\section{Research Theoretical Framework}

This figure shows the framework of the proposed pillars of the research project that will be investigated. It embraces five essential components; HTA conceptualization, implementation, capacity, gaps, and strengthening strategies, as they are clearly covered in the objectives section.

\section{Research Aim And Objectives}

Given the World Bank income classification, the selected countries were classified as follows: Canada and Switzerland as high-income countries, Lebanon as an upper-middle-income country, Palestine as a lower-middle-income country, and Tanzania as a low-income country (see table one in appendices). The overall aim of this research is to comprehensively understand the HTA systems and evaluate the current technologies of the HSs using HTA in these five countries through the following specific objectives:

- Assess the understanding level among stakeholders on HTA concept, importance, and practices;

- Explore the HTA applicability, capacities, and utilization in the health decision and policy-making process;

- Evaluate the current health technologies and interventions using HTA;

- Identify gaps and propose feasible solutions such as a framework to support best practices for HTA and knowledge translation strategies at the national and regional arenas.

\section{Research Question(S) And/or Hypothesis(Es)}

Page 3/11 
The research project is expected to answer the following questions:

- Is the concept, functions, and practices of HTA understood by HTA stakeholders in selected countries?. The study assumed that there is a varying and inadequate level of conceptualization and knowledge about HTA and this hinders its strengthening in the health sectors.

- Is the implementation of health technologies and interventions managed and applied appropriately, evaluated systematically, and actively used in the decision-making and planning process?, and are HTA systems in the five settings, mainly in developing countries, are capacitated and resourced enough?. The hypothesis is that technologies and interventions are unsystematically or might be inappropriately applied and evaluated as HTA is non-institutionalized and used in health decision-making, especially in middle and low-income countries. HTA capacity and resources are varied and exit but misapplied and misallocated, largely in developing countries.

- Are there differences between the five contexts in terms of HTA knowledge level, application, capacity, barriers, and current strategies?. There is an assumption that there are significant differences and countries may share the same challenges and different practices related to HTA aspects.

- Will the strengthening framework or roadmap fit the five contexts and other similar countries and lead to achievable results?. It is assumed that the framework may vary from one environment to another with the belief that this framework will make the advancement of practices, strategies, and interventions possible if HTA is appropriately acknowledged, embraced and applied.

\section{Methodology}

\subsection{Study design}

The research project is a cross-sectional study and will apply mixed methods; quantitative and qualitative methods along with scoping review to address these three dimensions: 1) the conceptual dimension of HTA concepts, functions and usage, 2) the technical and functional dimension in terms of HTA applicability and capacity, and 3) gaps and solutions identification for HTA institutionalization and strengthening.

\subsection{Study setting / data sources}

The uniqueness that the study will be conducted in six different countries selected based on the World Bank income classification; Canada, Switzerland, Jordan, Lebanon, Palestine, and Tanzania to figure out differences, similarities, gaps, successes, and lessons learned among these settings. In each country, fifty semi-structured questionnaires, twenty in-depth interviews, and one national focus group discussion will be conducted with health experts, managers, and policymakers selected purposively from the 1 st and 2 nd levels of the HS structure and its departments. Thus, intensive consultations to sort out comprehensive participants and participating list based on pre-defined criteria and conditions will be approached. The participants from the six settings will be mainly invited from these top levels of the government sector (Ministry of Health and relevant departments and ministries), academic, and private and nongovernmental organizations. Different sampling strategies will be used to maximize representation and knowledge saturation and reduce bias. Using scoping review, data from secondary sources will be used such as published and unpolished national, regional, and international reports, briefs, articles, studies, facilities' records, and bulletins to complement the primary data. Pilot studies for the quantitative and qualitative tools will be consulted with peers and tested in the field.

\subsection{Study population}

All health experts, including healthcare managers, and policymakers working in various relevant departments in the HS in each country are the study population. All from the first (management) and second (technical) levels of the three health sectors, forms the HS in each country, should be officially occupying whether management or technical level. Their names will be sorted in a comprehensive list through peer reviews and consultations then a subset of this list will be selected in probability and non-probability sampling way using different sampling strategies. A simple sample will be used to select fifty technical participants from the identified list, the second level of the HS, to answer fifty semi-structured questionnaires. Participants from the first level will be recruited through the critical case, snowballing, and convenience non-probability sampling process to participate in two instruments: twenty in-depth interviews, and one national focus group discussion.

\subsection{Sample size (sample size assumptions / estimate)}


The number of health experts, managers, and policymakers from both gender and structural levels in each country likely to be one hundred. Fifty technical participants will participate in filling questionnaires, twenty from the front line of the HS will invite to twenty indepth interviews, and lastly, 6 to 9 participants mixed from the management and technical levels of the HS will participate in one national workshop.

\subsection{Sampling method and inclusion and exclusion criteria}

In each country, we will select fifty participants for completing the questionnaire, twenty key-informants for interviews, and 6-8 participants for one focus group discussion. Selection will consider inviting participants from both genders from the HS. To reach a good representation, we will use various sampling strategies to select participants in probability and non-probability approaches from different levels of the HS structure in each country (management and technical levels). Selection criteria will be put after peer consultations. A comprehensive list through the peer reviews and consultations will be formed. Using a useful probability and nonprobability sampling way will support reaching a large representative sample through various strategies. Simple sampling will be used to select fifty technical participants from the identified list, the second level of the HS, to answer fifty semi-structured questionnaires. Participants from the first level will be recruited through the critical case, snowballing, and convenience non-probability sampling process to participate in two instruments: twenty in-depth interviews, and one national focus group discussion.

\subsection{Data collection (data collection method(s) and tool(s)}

This research will use fours tools as follows:

1. Scoping review by conducting a quick review to extract relevant published and unpublished local, regional, and international relevant resources such as governments and institutions websites, articles and magazines, scientific repositories and databases, and knowledge platforms.

2. Fifty semi-structured questionnaires will be administered to fifty technical participants from the HS in each country. The near-final adapted questionnaire that was developed by the WHO is supplemented with this proposal.

3. Twenty in-depth interviews with key informants from the country HS.

4. One national focus group discussion workshop consists of 6-8 persons from policy and technical levels of the HS in each country will be also carried out.

These tools whether quantitative and qualitative methods along with scoping review will be designed to address these three dimensions: 1) the conceptual dimension of HTA concepts, functions and usage, 2) the technical and functional dimension in terms of HTA applicability and capacity, and 3) gaps and solutions identification for HTA institutionalization and strengthening. To make this feasible, a specific plan with a defined timeline, instructions, training, roles, names, and expected deliverables will be constructed to guide the collection data measures. This will be performed by data collectors, one from each country, under close guidance and follow up of the $\mathrm{PI}$ and the co-investigators.

\subsection{Data management plan}

Specific criteria, such as identifying sources, relevant materials, and aspects to focus on, to search resources will be defined to conduct a meaningful scoping review. Other tools such as questionnaires, interviews, and focus group discussion questions will be constructed based on previous literature reviews such as recommended theoretical and technical frameworks, approaches, tools, studies, and experiential cases on the HTA. Data from three instruments will be stored and protected in a secured file with limited accessibility of the PI. Quantitative data will be managed and analyzed by the IBM Statistical Package for the Social Sciences (SPSS) while qualitative data, MAXQDA 12 (VERBI GmbH, Berlin), a software package for qualitative data management and analysis will be used. Lastly, an Excel program to categorize and analyze data from the scoping review will be used. Descriptive and inferential quantitative analysis and thematic qualitative analysis will be conducted using the abovementioned software programs. The methodological approach of this research was built based on similar reliable international studies (Lessa and Ferraz, 2017; Oortwijn et al., 2013; Tantivess et al., 2017; WHO, 2017).

\subsection{Coordination, monitoring and quality control plan}

Three essential coordination and quality assurance will be followed to make the fieldwork successful and feasible:

1. Strong collaboration is already established with partners and collaborators from the Middle East countries such as Philadelphia University in Jordan, the American University of Beirut in Lebanon, Najah National University in Palestine, Ifakara Health Institute (IHI) 
in Tanzania. In Europe, an established network with the Swiss Tropical and Public Health Institute in Switzerland could be the potential collaborating institution. Having an existing collaboration with McGill University in Canada could also a catalyst to perform the data collection properly. This broad well-established network will support this research intervention and fieldwork in particular in three purposes: 1) providing useful scientific and technical consultations, 2) facilitating all country fieldwork, and 3) assisting in knowledge translational activities. Having such inter-country, regional, and international networks and outstanding research and advisory teams, this research project will be a reality and it will exactly meet the stated goals.

2. Solid fieldwork consists of specific activities, timeline, responsible persons, roles, and expected outcomes and indicators for the four tools will be established before the implementation of a project to measure the work progress, achievement, and deliverables. In addition to the research team led by the $\mathrm{PI}$, one well-trained and specialized data collector or assistant from each country will be recruited. One training and coaching session will be conducted to train data collectors and assistants on the whole process of the project and providing them specific technical techniques and skills.

3. Data collection process, starting from search strategies and building tools, will be strictly governed by a mixed scientific team from all partners and settings in a collaboration with the $\mathrm{PI}$ and co-investigators. This is to collaborate in building robust strategies and tools, ensuring appropriate data collection, guarantying good data management and protection, and then designing appropriate and standardized data analysis approaches based on clear indicators and conditions developed from consultations and scientific evidence.

\subsection{Ethical considerations}

This research deals with experts, policymakers, and managers, and administrative approval is essential will be taken from the health authorities from each country to access the institutions to implement the activities. In this research, there is no human subjects' experimentation or trials to obtain ethical approvals. However, two kind of ethical approvals from countries were obtained in 2020, particularly from the national Helsinki ethics committee in Palestine (number PHRC/HC/732/20) and the institutional review board of Philadelphia University in Jordan. Copies of both approvals are attached in the supplements. Further approvals from other implementing and collaborating institutions may requested. An official letter on the research along with project summary and documents will be submitted to the administrations of the participating institutions in the six countries to receive their administrative approval. The PI and team members will initially contact potential participants to provide them with a copy of the study information sheet to get their individual consent form. The expected limitations of obtaining the ethical, administrative, and individual approvals can be the time-consuming. This can be managed in the earlier before the stage of project implementation through receiving support from the partners and systematic communications and using the WHO ethical and administrative official approvals to be submitted along with the project documents. Lastly, as mentioned earlier, the confidentiality of participants' perceptions and data will be anonymously classified, appropriately stored, and carefully secured in one laptop according to the WHO guidelines with a proper security and accessibility measure under the PI responsibility.

\section{Discussion}

HTA has been hypothesized to play a significant role in improving healthcare delivery and universal health coverage through developing reliable evidence, insights, and comparisons to be added to the current knowledge advancement and for innovating further global and regional best practices of HTA. This study will provide a comprehensive understanding about the HTA systems in different income levels countries' settings. This study will evaluate the current technologies of the HSs, identify gaps and propose feasible solutions to support best practices for HTA and knowledge translation strategies at the national and regional arenas.

To our knowledge, this will be the first study on HTA conducting in six different countries selected based on the World Bank income classification; Canada, Switzerland, Jordan, Lebanon, Palestine, and Tanzania. It is expected to produce a thorogh evidence-based framework highlighting the differences, similarities, gaps, successes, and lessons learned among these settings. The study will encourage the state's officials to make serious decisions for strengthening HTA at national and regional levels to strengthen the resilience and capacity of health systems in developing countries specifically in emergency settings to respond effectively during pandemics.

The study design will engage health experts, managers, and policymakers from the six settings from both gender and structural levels in each country including levels of the government sector (Ministry of Health and relevant departments and ministries), academic, and 
private and non-governmental organizations. The mixed participants from the management and technical levels of the HS will lead to better professional representation, inclusive different mindsets, and discipline to effective HTA implementation.

Findings from this study will inform health service planners and policymakers to strengthen the practice of HTA during decisionmaking, resource allocation, protection and prevention, detection and diagnostics, and therapeutics. Thus, its implementation is expected to improve health surveillance, HS management, health education, and clinical decision-making, and to support behavioral changes related to public-health priorities and long-term disease management.

The study may face limitations related to obtaining the ethical, administrative, and individual approvals, which can be timeconsuming. This will be managed in the earlier before the stage of project implementation through receiving support from the partners and systematic communications and using the WHO ethical and administrative official approvals to be submitted along with the project documents. The spread of COVID-19 pandemic and infection risks might limit the movement and face to face interviews. This can be mitigated through using the virtual remote communication. In addition, some potential challenges of the preoccupation of HS actors from the front line management in low and middle low-income countries. This can be managed by engaging $1 \mathrm{st}$ and $2 \mathrm{nd}$ levels of the HS structure in each country in the assessment.

In terms of HTA implementation study, coordination and quality control is a key to success. We are aware that time constraints and workload of each stage may vary, but we are confident that intense communication during the HTA tools application, monitoring and continuous discussions could improve participants' contribution in this study. Zero potential biases will be guaranteed due to the various HTA scientific data collection tools (interviews, FGDs, and questionnaires), and the contribution of the 1st and 2nd levels of the HS structure in each of the sixth countries.

\section{Implications}

This research yields tremendous direct and indirect benefits where its outputs target various beneficiaries, groups, and stakeholders at the local, regional, and global levels in the short or long term on the field of HTA.

The project has two kind of beneficiaries that will be benefiting from the outcomes of the implementation of the project; direct and indirect beneficiaries. Direct will be: 1) policymakers, decisionmakers, health planners, health officials, thinkers, experts, and strategists exist in the national HS in each county, 2) heads, managers, and directors of various public health departments, such as planning, public health and epidemiology, primary healthcare, hospital, health economic, health information, and pharmaceuticals and supplies, 3) directors and representatives of non-state actors and organizations such as private sector, non-governmental organizations, national institutions, and community-based organizations, 4) scientific community and researchers for further research attempts. The main benefit for this group, number four, is assisting the first three beneficiaries in the existing strategies and plans and providing them best practices and feasible solutions. While, beneficiaries who will be indirectly benefiting are population and people (healthcare consumers) in the six countries in particular and other many countries sharing the same context and classification of the countries targeted in this study. This indirect benefit could be tangible in the long-term by enjoying sufficient level of healthcare accessibility, coverage, equity, affordability, and quality if the HTA best practices appropriately applied and institutionalized.

There are significant contributions and outcomes expected to be accomplished from this research as follows:

- Advance an innovative triangulated analytic framework to be applied in different contexts and a comparative analysis of the five different settings, including Canada and Switzerland, will be introduced to enrich the national and international knowledge libraries and to complement the gap in light of literature limitations.

- The research findings, reinforced by the Canadian and Swiss HTA models, can be a benchmark for the countries for better strategies and tools for HTA systems.

- Further specific assessments are needed to investigate HTA management and methodology in the developed countries like Canada and Switzerland despite the plenty of literature (Dankó, 2014; Juan E. del Llano-Señarís and Carlos Campillo-Artero, 2015; Tantivess et al., 2017; WHO, 2017). In contrast in developing countries, as deep contextual assessments are still needed, for instance, HTA was not examined in Palestine and insufficiently investigated in Lebanon and Tanzania.

- Knowledge production and translation: 4 papers will be published in a prestigious journal, a book on HTA will be designed for research \& education purposes, 5 dissemination workshops will be held, HTA Webinars \& learning platform will be established, and policy briefs will be produced. 
About contribution to the relevant scholarly literature, there is an abundance of literature covering the subject of HTA in developed countries. In contrast, the literature on HTA in middle and low-income countries still needs further deep contextual assessments to reflect a clearer landscape of HTA. Most of these countries have utmost necessity for HTA in their HSs owing to the scarcity of resources and the specificity of challenges. For instance, HTA did not examine in Palestine, and at the same time, it is insufficiently investigated at the country and regional levels of Lebanon and Tanzania. Therefore, this research project seeks to fill this knowledge gap through updating and advancing novel knowledge and evidence to inform both communities, scientific and decision making, in selected countries. This evidence can assist scientists to build supplementary knowledge and can encourage the state's officials to make serious decisions for strengthening HTA at national and regional levels. It is time for adopting new useful and interdisciplinary approaches such as HTA to strengthen the resilience and capacity of health systems in developing countries to respond effectively during pandemics.

\section{Conclusion}

This research will generate optimal knowledge translation strategies for useful HTA frameworks and models benefiting mainly the low and least developed settings through adopting HTA as an essential approach to improve HSs and tackle existing challenges, particularly in developing countries as they share most of the health burdens in our region and worldwide.

The research will form cutting-edge evidence and reference not only for the six countries, but also for the global, regional, and national endeavors with regards to opening a room for HTA best application and optimization based on the produced knowledge from this research. It will reveal lessons learned, determine gaps, and set an applicable strengthening framework for HTA. This framework will eventually aid the decision and policymakers in these countries, and other similar countries and international organizations to build a well-enabled and institutionalized HTA for better universal health coverage, health systems, and multi-sectoral development.

\section{Declarations}

\section{Ethics approval and consent to participate}

This research deals with experts, policymakers, and managers, and administrative approval is essential will be taken from the health authorities from each country to access the institutions to implement the activities. In this research, there is no human subjects' experimentation or trials to obtain ethical approvals. However, two kind of ethical approvals from countries were obtained in 2020, particularly from the national Helsinki ethics committee in Palestine (number PHRC/HC/732/20) and the institutional review board of Philadelphia University in Jordan. Copies of both approvals are attached in the supplements. Further approvals from other implementing and collaborating institutions may requested. An official letter on the research along with project summary and documents will be submitted to the administrations of the participating institutions in the six countries to receive their administrative approval. The PI and team members will initially contact potential participants to provide them with a copy of the study information sheet to get their individual consent form. The expected limitations of obtaining the ethical, administrative, and individual approvals can be the time-consuming. This can be managed in the earlier before the stage of project implementation through receiving support from the partners and systematic communications and using the WHO ethical and administrative official approvals to be submitted along with the project documents. Lastly, as mentioned earlier, the confidentiality of participants' perceptions and data will be anonymously classified, appropriately stored, and carefully secured in one laptop according to the WHO guidelines with a proper security and accessibility measure under the PI responsibility.

\section{Consent for publication}

Not applicable

\section{Availability of data and material}

To keep database protected, data from the participants through questionnaire, interviews, and FGDs will be saved confidential under unanimous classification in the principal investigator official laptop. Confidentiality and anonymity will be also applied when coded data are published. These data are stored in a highly secured laptop with a secured key file entry, under the control of the principal investigator (MK) and the supervisor (SA), and only they have the right of accessibility to review and use these data through a secure institutional system. 


\section{Funding}

All sources of funding for the research and the roles of the funding body, if exist, will be declared in the manuscripts that will be generated from the research. No funding awarded for this project so far but the research team attempting to attract funding.

\section{Competing interests}

The authors declare that they have no competing interests.

\section{Authors' contributions}

$\mathrm{SA}, \mathrm{MT}$, and MK contributed to the conception and methodological design. MK contributed to the writing of the project proposal. MK and $A B$ have developed the tools of the research through conducting extensive and focused related literature reviews. MK and $A B$ will be handling data collection, management, analysis, and reporting results. All authors mentioned read and approved the final version of the proposal.

\section{Acknowledgements}

This research received support and assistance from professors and experts from various international institutions. We show special gratitude to the following professors and experts who reviewed the proposal and put their useful scientific advice and made additional enrichment insights. The experts are as follows: Prof. Kaspar Wyss, Director of Swiss Centre for International Health in Switzerland; Prof. Yehia Abed, Al-Quds University, Palestine; Dr. Hamza Abdeljawad, Al-Quds University, Palestine; and Dr. Abdulsalam Khayyat, AnNajah National University, Palestine.

\section{References}

AlKhaldi, M., 2018. Palestinian Health Research System: Moving Forward (Ph.D. Thesis). University_of_Basel.

Banta, D., 2003. The development of health technology assessment. Health Policy 63, 121-132.

Dankó, D., 2014. Health technology assessment in middle-income countries: recommendations for a balanced assessment system. J. Mark. Access Health Policy 2, 23181. https://doi.org/10.3402/jmahp.v2.23181

HTA in Switzerland - SwissHTA - Swiss Health Technology Assessment [WWW Document], n.d. URL http://www.swisshta.org/index.php/HTA_in_Switzerland.html (accessed 2.24.19).

Juan E. del Llano-Señarís, Carlos Campillo-Artero, 2015. Health technology assessment and health policy today: a multifaceted view of their unstable crossroads. Springer Berlin Heidelberg, New York, NY.

Lessa, F., Ferraz, M.B., 2017. Health technology assessment: the process in Brazil. Rev. Panam. Salud Pública 41, e25.

Marcial Velasco Garrido, Finn Børlum Kristensen, Camilla Palmhøj Nielsen, Reinhard Busse, 2008. Health technology assessment and health policy-making in Europe: current status, challenges, and potential, Observatory studies series. World Health Organization on behalf of the European Observatory on Health Systems and Policies, Copenhagen.

Menon, D., Stafinski, T., 2009. Health Technology Assessment in Canada: 20 Years Strong? Value Health 12, S14-S19.

https://doi.org/10.1111/j.1524-4733.2009.00554.x

The Middle East and North Africa Health Policy Forum, 2017. Health Technology Assessment (HTA) for Universal Health Coverage. Presented at the MENA HPF REGIONAL FORUM, Cairo, Egypt.

Oortwijn, W., Broos, P., Vondeling, H., Banta, D., Todorova, L., 2013. MAPPING OF HEALTH TECHNOLOGY ASSESSMENT IN SELECTED COUNTRIES. Int. J. Technol. Assess. Health Care 29, 424-434. https://doi.org/10.1017/S0266462313000469

Oortwijn, W., Mathijssen, J., Banta, D., 2010. The role of health technology assessment on pharmaceutical reimbursement in selected middle-income countries. Health Policy 95, 174-184. https://doi.org/10.1016/j.healthpol.2009.12.008 
Ritrovato, M., Faggiano, F.C., Tedesco, G., Derrico, P., 2015. Decision-Oriented Health Technology Assessment: One Step Forward in Supporting the Decision-Making Process in Hospitals. Value Health 18, 505-511. https://doi.org/10.1016/j.jval.2015.02.002

Rugunda, R., Asadi-Lari, M., 2014. Fourth report of Committee B.

Tantivess, S., Chalkidou, K., Tritasavit, N., Teerawattananon, Y., 2017. Health Technology Assessment capacity development in low- and middle-income countries: Experiences from the international units of HITAP and NICE. F1000Research 6.

https://doi.org/10.12688/f1000research.13180.1

WHO, 2017. Global Survey on Biomedical Engineering Professionals in Health Technology Assessment. Springer, pp. 595-598.

WHO, 2015. 2015 Global Survey on Health Technology Assessment by National Authorities. Main findings.

WHO, 2013. SEA/RC66/R4-Health intervention and technology assessment in support of universal health coverage.

WHO, EMRO, 2014. Health technology assessment: a tool for informed decision-making.

WHO, EMRO, n.d. RC66 | Evidence-informed policy-making for health.

WHO, EMRO n.d., n.d. WHO EMRO | Health technology assessment: a tool for informed decision-making | RC61 | About WHO [WWW Document]. URL http://www.emro.who.int/about-who/rc61/health-technology-assessment.html (accessed 12.10.18).

WHO, EMRO, Cairo, 2015. RC62_2015_Presentation_Technical_Meeting_Health_Technology_Assessment_16603_EN.pdf [WWW Document]. URL

http://applications.emro.who.int/docs/RC62_2015_Presentation_Technical_Meeting_Health_Technology_Assessment_16603_EN.PDF? ua=1 (accessed 11.8.19).

\section{Figures}

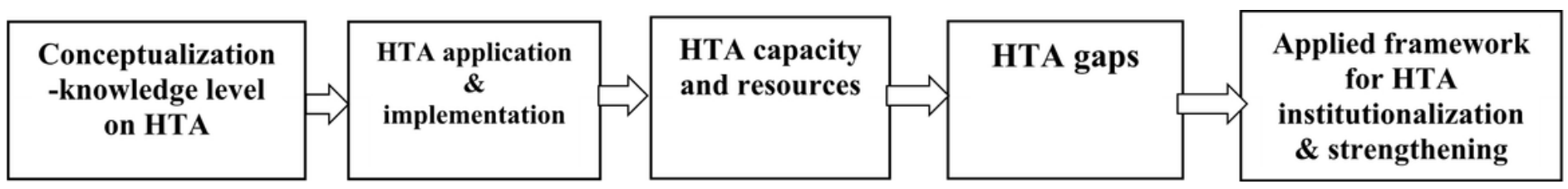

Figure 1

This figure shows the framework of the proposed pillars of the research project that will be investigated.

\begin{tabular}{|c|}
$\begin{array}{c}\text { Conceptualization } \\
\text {-knowledge level } \\
\text { on HTA }\end{array}$ \\
HT
\end{tabular}

Figure 1

This figure shows the framework of the proposed pillars of the research project that will be investigated.

\section{Supplementary Files}

This is a list of supplementary files associated with this preprint. Click to download.

- HTASURVEYENGLISH1FINAL1.docx

- HTASURVEYENGLISH1FINAL1.docx

- HTAprojectsupplements.docx

- HTAprojectsupplements.docx 
Page 11/11 